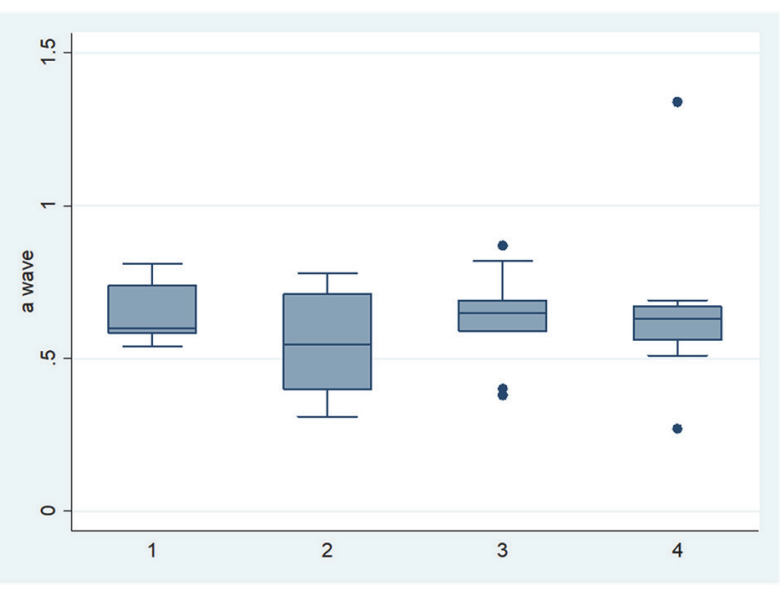

Abstract 10 Figure 2 Boxplot showing A wave velocities by LA scar burden in quartiles

velocities $(\mathrm{r}=0.26, \mathrm{p}=0.11)$ or $\mathrm{E}: \mathrm{A}$ ratio $(\mathrm{r}=-0.02, \mathrm{p}=0.91)$ (figure 2). A significant correlation between A wave velocity and CHADS2VASc was observed $(r=0.49, p=0.001)$.

Conclusion Our study demonstrates no relationship between degree of LA scarring and reduced LA function on TTE as assessed by the A wave. It has been established that structural remodeling in $\mathrm{AF}$ (such as atrial dilatation) may occur independently of the electrical remodelling. A potential explanation for our findings is that the electrical scarring in AF, which results in alterations in refractory periods, precedes the negative remodeling which ultimately results in reduced atrial function. This hypothesis would need to be further evaluated in larger studies.

\section{TEN-YEAR CLINICAL OUTCOMES FROM A RANDOMIZED TRIAL OF POLYMER-FREE VERSUS DURABLE POLYMER DRUG-ELUTING STENTS TEN-YEAR RESULTS OF THE INTRACORONARY STENTING AND ANGIOGRAPHIC RESULTS: TEST EFFICACY OF SIROLIMUS- AND PROBUCOL- AND ZOTAROLIMUS- ELUTING STENTS (ISAR-TEST 5) TRIAL}

1,2RA Byrne, ${ }^{3} \mathrm{~S}$ Kufner, ${ }^{3} \mathrm{M}$ Ernst, 1,3 $\mathrm{R}$ Colleran, ${ }^{3} \mathrm{~A}$ Kastat. ${ }^{1} \mathrm{C}$ adionascular Research Institute (CVRI) Dublin, Mater Private Hospital, Dublin, Ireland; '2School of Pharmacy and Bimolecular Sciences, RCSI University of Medicine and Health Sciences, Dublin, Ireland; ${ }^{3}$ Department of Cardiovascular Diseases, German Heart Centre, Munich, Germany

10.1136/heartjnl-2020-ICS.11

Background Outcome data after extended long-term follow-up of patients with coronary artery disease treated with drug-eluting stents (DES) in randomized clinical trials is scant.

Objective Performance differences between devices may be expected to emerge over time depending on whether stenting is done with polymer-free or durable polymer DES. We assessed the 10-year outcomes of patients enrolled in the ISAR-TEST 5 trial.

Methods 3002 patients were randomized to treatment with either polymer-free sirolimus- and probucol-eluting stents (PFSES) $(n=2002)$ or durable polymer zotarolimus-eluting stents (DP-ZES) $(n=1000)$. The primary endpoint was the composite of cardiac death, target vessel-related myocardial infarction or target lesion revascularisation (a device-oriented composite endpoint, DOCE). Additional endpoints of interest were the patient-oriented composite endpoint (POCE), including allcause death, any myocardial infarction or any revascularisation, individual components of the composite endpoints, and definite or probable stent thrombosis.

Results The median age of the patients at randomization was 67.8 years. At 10 -years, $63.9 \%$ of patients were alive. The rates of DOCE and POCE were high in both groups with no difference in the incidence between PF-SES and DP-ZES, (DOCE: $43.8 \%$ versus $43.0 \%$, respectively, hazard ratio = 1.01, 95\% CI, 0.89-1.14; $\mathrm{P}=0.90$ ), (POCE: 66.2\% versus $67.7 \%$, respectively, hazard ratio $=0.94,95 \% \mathrm{CI}, 0.86-1.04$; $\mathrm{P}=0.22$ ). The rates of the individual components of the composite endpoints were comparable in both groups. The incidence of definite/probable stent thrombosis over 10 years was low and comparable in both groups $(1.6 \%$ vs. $1.9 \%$; hazard ratio $=0.85$ [95\% CI, 0.46-1.54], $\mathrm{P}=0.58)$.

Conclusion At 10 years there were no measurable differences in outcomes between patients treated with polymer-free versus durable polymer DES. The incidence of stent thrombosis was low and comparable in both groups. High overall adverse clinical event rates were observed during extended follow-up.

\section{ELECTROCARDIOGRAPHY PREDICTORS FOR PACEMAKER INSERTION POST TAVR}

D Ranganathan, B O'Sullivan, E McAuliffe, SM O' Connor, N Rady, F Fahy, P Kearney. Cork University Hospital, Cork, Ireland

\subsection{6/heartjnl-2020-ICS.12}

Introduction The development of Transcatheter Aortic Valve Replacement (TAVR) has provided an alternative to surgical valve replacement in high-risk population. Post-procedural length of stay is one of the main cost components after TAVR and is significantly influenced by prolonged monitoring for new conduction disturbances. Occurrence of advanced conduction delays, if left untreated, can be responsible for sudden cardiac death after discharge.

Objective We aimed to determine the electrocardiographic predictors of advanced conduction disturbance that required a permanent pacemaker implantation (PPM) after TAVR.

Methods All consecutive patients who underwent TAVR between January 2016 to July 2019 were identified from by a retrospective review of TAVR database at this centre.

Results A total of 77 patients underwent TAVR within the study period and 7 patients were excluded as they had a preexisting pacemaker/implanted cardiac defibrillator and 2 patients were excluded due to insufficient data. 36 of the patients were males (52\%) with an average age of 82.1 years. The average BMI was 26.8 and 62 patients (91.1\%) were symptomatic with New York Heart Association class 3 or above.

All 68 patients had self-expanding Medtronic CoreValveTM. $10(14.7 \%)$ patients required a PPM within an average of 5.3 days for an indication of either complete heart block or intermittent complete heart block. Of the 10 patients who had a PPM inserted,3 patients had a Right Bundle Branch Block (RBBB) at baseline. These patients were all pacingdependent $(>80 \%$ pacing when lower rate was programmed at 60 beats $/ \mathrm{min})$ at 6 weeks post-implantation follow-up. 4 out of 7 patients $(57 \%)$ who had a non-RBBB pattern (i.e. LBBB or normal QRS complex) at baseline had an average pacing requirement of $<2 \%$ at 6 weeks post implant follow- 